\title{
Surgical management of skin cancers: Experience from a regional cancer centre in North India
}

\author{
Deo SV, Hazarika Sidhartha, Shukla Nootan K, Kumar Sunil, Kar Madhabananda, \\ Samaiya Atul \\ Department of Surgical Oncology, Institute Rotary Cancer Hospital, All India Institute of Medical \\ Sciences, New Delhi, India
}

Correspondence to: Dr. Deo SV, E-mail: svsdeo@yahoo.co.in

\section{Abstract}

AIMS: To review the disease profile and treatment outcome of patients with primary skin malignancies treated at a regional cancer centre. SETTINGS AND DESIGN: Surgical oncology unit of a tertiary care regional cancer centre. Evaluation of treatment outcome of patients with skin cancer from Surgical Oncology database was done. MATERIALS AND METHODS: Retrospective analysis of records of 77 patients with skin cancers treated between 1995 and 2002 was conducted. Profile of patients with skin cancer, surgical details including the management of primary tumour, regional lymph nodes and reconstructive procedures performed and survivals were analysed. STATISTICAL ANALYSIS: All computations were done using the Statistical Package for Social Sciences (SPSS-9). Descriptive statistics were calculated in a standard fashion and survival analysis was performed using Kaplan-Meier method. RESULTS: Skin cancers constituted 2.4\% (77/3154) of patients with cancer treated in the surgical oncology department. Squamous cell carcinoma (SCC) was the most common histological type $(55.8 \%)$ followed by melanoma (26.1\%) and basal cell carcinoma (BCC, 18.1\%). Forty one percent of patients had undergone some form of intervention elsewhere before being referred. Reconstruction was required in $55.8 \%$ patients with large postresection defects. Regional lymph nodal dissection was required in $32.4 \%$ of total patients. Five-year median disease-free survival for the entire study population was $75 \%$. CONCLUSIONS: Skin cancers constitute a small but significant proportion of patients with cancer. Unlike in the Western countries, SCC is the commonest histologic variety. Primary level inadequate intervention is very common. Optimal results can be obtained with radical surgery and optimal surgical margins along with a reconstructive procedure when needed.

Key words: Basal cell carcinoma, Malignant melanoma, Reconstruction, Skin cancers, Squamous cell carcinoma, Surgical margins

\section{Introduction}

Skin cancers are relatively uncommon malignancies worldwide, not ranking among the first ten common cancers. $^{[1]}$ There has been a progressive increase in the incidence of skin cancers, particularly that of cutaneous melanomas over the last few decades. ${ }^{[2]}$ Three most frequent primary skin cancers are basal cell carcinoma (BCC), squamous cell carcinoma (SCC) and malignant melanoma. Together SCC and BCC are referred to as nonmelanomatous skin cancers (NMSC).
In India, skin cancers constitute about $1-2 \%$ of all diagnosed cancers. Basal cell carcinoma is the commonest form of skin cancer worldwide, but various studies from India have consistently reported SCC as the most prevalent skin malignancy. ${ }^{[3]}$ Although complete data of incidence is not available, various cancer registries in India reported cumulative incidence of skin cancer varying from 0.5 to 2 per 100000 population. ${ }^{[4]}$ Although, the incidence of skin cancers in India is lower as compared to the Western world, because of a large population, absolute number of cases 
is estimated to be significant.

In spite of being a surface malignancy and more amenable not only to early detection, but also to a potential cure, treatment outcome and follow-up data about survival is scarce from the Indian population. In this study, we present the disease profile and treatment outcome of patients treated at a tertiary care regional cancer centre who were with these three primary skin malignancies; namely SCC, BCC and melanoma. Various surgical procedures carried out and the importance of reconstruction using skin grafts, local and regional flaps wherever required following radical excision of primary skin cancer, without compromising the recommended surgical margins has been highlighted.

\section{Materials and methods}

The computerised database of Surgical Oncology Department at a Regional Cancer Centre was reviewed retrospectively. Patient records of a total of 77 patients with skin malignancies, out of a total of 3154 surgically treated patients in the Department for various malignancies between 1995 and 2002 were analysed.

Patients were first evaluated in the out patient clinic by a team of Surgical and Radiation Oncologists and they underwent treatment according to the protocol decided by the same team. Apart from history and detailed clinical assessment, the pretreatment work up included a chest X-ray and routine blood examination. Imaging studies (CECT/MRI) were used only in locally advanced lesions to assess the extent of local infiltration. In patients with melanoma, ultrasound of the abdomen was used to evaluate any visceral or lymph nodal metastases. And in patients with enlarged regional lymph nodes, fine needle aspiration cytology (FNAC) was carried out to look for regional spread.

Operative procedures and width of surgical margins varied according to the histology and extent of local infiltration by the tumour. For BCC, local excision was carried out with a minimum margin of $0.5 \mathrm{~cm}$. For SCC, minimum three-dimensional margin taken was $1 \mathrm{~cm}$. For malignant melanoma, minimum margin taken was $2 \mathrm{~cm}$. Comprehensive regional lymph nodal dissection was performed if there was evidence of nodal involvement. Prophylactic lymph node dissection was not performed in any of the patients. For patients with skin cancers (SCC or Melanoma) of the extremities, where the disease had completely encased the neurovascular bundle or infiltrated bones, amputations were performed. In patients where excision was previously done elsewhere and if margin status was not clear, re-excision of the scar tissue and tumour bed was carried out. For large surgical defects not suitable for primary closure, reconstructions using split skin grafts, local (transposition/rotational advancement) or regional (fasciocutaneous/myocutaneous) flaps were performed. Adjuvant postoperative radiotherapy (50 Gy/25\# over 5 weeks) was given in cases of SCC/BCC with positive tumour margins, with locally advanced growths and in patients with positive nodal disease. After completion of treatment, all the patients were followed up at three monthly intervals to look for relapse of disease.

The collected database was analysed using SPSS-9 (SPSS Inc., Chicago, USA) statistical software. Disease free, survival analysis was performed using the KaplanMeier method.

\section{Results}

\section{Patient profile}

Skin cancers constituted $2.4 \%(77 / 3154)$ of patients treated in the Surgical Oncology Department between 1995 and 2002. Out of these, 43 patients (55.8\%) had SCC, 20 patients $(26.1 \%)$ had cutaneous malignant melanoma and 14 patients (18.1\%) had BCC. Fifty patients were men and the remaining 27 were women (M:F $1.8: 1)$. Mean age of the entire study population was 51.8 years (range $18-87$ years). Thirtytwo patients $(41.5 \%)$ had undergone some form of intervention before being referred, with inadequate surgical resection being the most common intervention. Eight patients received radiotherapy outside and were referred because of incomplete response or development of recurrence.

Distribution of primary sites involved is shown in Table 1. Forty-two percent of patients had locally advanced growths with involvement of deeper underlying structures such as subcutaneous tissue, muscle, bone and neurovascular bundle. Twenty-five patients had regional lymph nodal involvement. Fourteen out of 43 patients with SCC (32.5\%) and 11 out of $20(55 \%)$ patients with melanoma required regional lymph nodal dissection.

\begin{tabular}{lcc}
\hline \multicolumn{3}{l}{ Table 1: Tumor with tumors $\left({ }^{*} n=77\right)$} \\
\hline Sites & No & $\%$ \\
\hline Scalp and face & 31 & 40.2 \\
\hline Lower extremities & 28 & 36.4 \\
\hline Upper extremities & 6 & 7.8 \\
\hline Trunk & 6 & 7.8 \\
\hline Inguino-scrotal & 6 & 7.8 \\
\hline${ }^{*} n=$ total number of patients & & \\
\hline
\end{tabular}




\section{Treatment details}

Types of surgical procedures carried out are described in Table 2. Eight patients $(10.4 \%)$ required limb amputation because of the involvement of neurovascular bundle or bone. Primary closure was possible in 26 patients $(33.7 \%)$. Remaining 43 patients $(55.8 \%)$ with large post resection defects required some form of reconstruction. Out of these, skin graft was used in 15 patients $(34.9 \%)$. In the remaining 28 patients (65.1\%), reconstructions using various flaps [Table 2] were carried out. Adjuvant radiotherapy to local and/or regional sites was used in 21 patients $(27.2 \%)$.

\section{Morbidity and mortality}

There were no intraoperative or postoperative deaths. Immediate morbidity in the form of wound infection, partial flap necrosis, loss of skin grafts occurred in 12 patients $(15.5 \%)$. All the complications were managed conservatively. None of the patients required resurgery.

\section{Histopathology}

Mean size of tumour was $2.85 \mathrm{~cm}$ (range: 0-16). Three patients (BCC-2, Melanoma-1) had positive tumour margins. Among the patients undergoing regional lymph nodal dissection, 24 out of 25 patients had tumour detected in the nodal specimens. The mean number of lymph nodes harvested from dissected specimen was 10.8 (range: 3-31). The mean number of positive nodes was 2.97 (range: $0-11$ ). Twenty out of 24 patients $(83.3 \%)$ with prior surgical intervention elsewhere, had residual tumour identified in the pathology specimen.

\begin{tabular}{lc}
\hline Table 2: Types of surgical procedures $\left({ }^{*} n=77\right)$ \\
\hline Surgical procedures & $n$ \\
\hline Wide excisions & 18 \\
\hline Simple & 15 \\
\hline With skin grafting & 28 \\
\hline With flaps & 3 \\
\hline Amputations & 10 \\
\hline Minor & 3 \\
\hline Major & 25 \\
\hline Orbital exenteration & 15 \\
\hline Lymph nodal dissection & 11 \\
\hline Reconstructions $(n=43)$ & 17 \\
\hline Split skin graft & \\
\hline Local (transposition/rotational advancement) flaps \\
\hline Regional (fasciocutaneous/myocutaneous) flaps \\
\hline${ }^{*} n=$ number of patients
\end{tabular}

\section{Follow up and survival}

Median follow up period was 16.4 months (range: 191 months). During follow-up ten out of 77 (12.9\%) patients had disease relapse. Recurrence pattern is shown in Table 3. Subgroup analysis revealed a significantly high relapse rate $(25 \%)$ in patients with melanoma. Out of the three patients with positive tumour margins after excision at our centre, two with BCC were alive and disease free and the one patient with melanoma had developed systemic relapse and died due to the disease. The median time for developing recurrence was 13.9 months (range: 3-74 months). On Kaplan-Meier survival analysis, 5-year median diseasefree survival in the study population was $75 \%$. As shown in Figure 1, 5-year disease-free survival for BCC was $100 \%$, for SCC was $82 \%$ and for melanoma was $52 \%$. At our last update, 63 patients $(81.8 \%)$ were alive and disease free, eight patients (10.3\%) were alive with disease and six patients $(7.9 \%)$ were reported to be dead

\section{Discussion}

Various reports from India show wide variations in the

\begin{tabular}{|c|c|c|c|}
\hline $\begin{array}{l}\text { Table 3: Types of relapse } \\
\text { cancers }\end{array}$ & thr & types & skin \\
\hline $\begin{array}{l}\text { Skin cancer type (number of } \\
\text { patients relapsed/total number } \\
\text { of patients) }\end{array}$ & & lapse pat & \\
\hline & Local & Regional & Systemic \\
\hline $\mathrm{BCC}(0 / 14)$ & 0 & 0 & 0 \\
\hline $\operatorname{SCC}(5 / 43)$ & 1 & 4 & 0 \\
\hline Melanoma $(5 / 20)$ & 1 & 4 & 4 \\
\hline
\end{tabular}

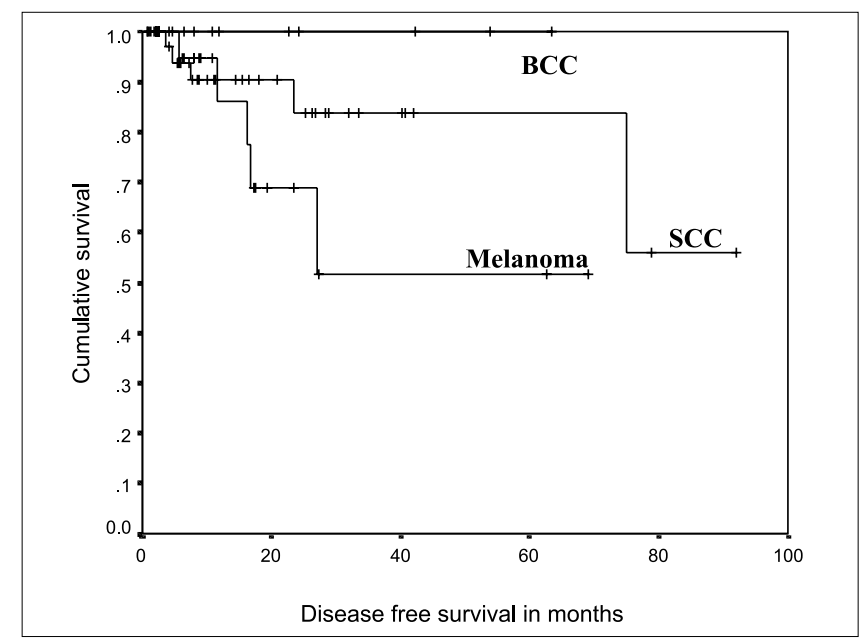

Figure 1: Disease-free survival curve for BCC, SCC and melanoma 
incidence and types of skin cancer from different parts of the country. Neve first reported 'Kangri' cancer in the Kashmir region in 1900. Khanolkar and Suryabhai described a new type of skin cancer - 'Dhoti cancer' in 1945. ${ }^{[5]}$ Very few literatures have been published from India in the recent period. ${ }^{[6]}$

The reported incidence of skin cancers in India is less than $1 \%$ of all cancers. ${ }^{[7]}$ In this study, common skin cancers constituted $2.4 \%$ of patients surgically treated at a referral regional cancer centre. Although there has been no significant increasing trend in the age-adjusted incidence of skin cancers in Indian patients, but in the Western countries, the incidence of skin cancers, especially of malignant melanoma has been rising for the past 40 years. ${ }^{[8,9]}$ In the Western countries, a vast majority of skin cancers are NMSC, mainly BCC. ${ }^{[10,11]}$ However similar to various earlier studies from India, ${ }^{[5,12]}$ the current study also shows that the most common histologic type of skin cancers is SCC $(55.8 \%)$.

The biologic behaviour of skin malignancies varies widely. Basal cell carcinoma rarely metastasizes and has an excellent prognosis and survival, whereas melanoma can be one of the most lethal malignancies with a high propensity for regional and systemic spread. Squamous cell carcinoma has $2-6 \%$ incidence of distant metastasis. ${ }^{[13]}$ Approximately 3-10\% of patients with melanoma present with metastatic disease in the absence of a clinically demonstrated primary lesion. ${ }^{[14]}$
Basel cell carcinoma usually occurs on sun-exposed areas [Figure 2] and SCC most commonly appears on sundamaged skin. Squamous cell carcinoma can also arise in the precursor lesions such as actinic keratoses, Bowen's disease, leukoplakia, or chronic scars. ${ }^{[15]}$ Melanoma arises most commonly on the skin of the back in men and on the lower extremities in women in Western countries, ${ }^{[10]}$ whereas sole of foot is the most common site for melanoma among Indian patients [Figure 3]. ${ }^{[8,16]}$ Sole of foot was involved in $60 \%$ of patients with melanoma in the current study. However, the commonest site of involvement for all the three skin cancers in the current study was scalp and facial skin $(40.2 \%)$.

The most important investigation in a case of suspected skin cancer is a proper excisional biopsy. ${ }^{[17]}$. Because these tumours involve superficial structures most of these patients undergo inadequate surgical excision at primary care canters owing to the lack of awareness and surgical expertise. Inadequate clinical information pertaining to the stage of the disease and type of resection (size, depth and status of margins) may create dilemma about the need for resurgery at a referral centres. In the current study, 32 (41.5\%) patients had undergone some form of intervention elsewhere. Out of these, 24 patients had undergone resection of the primary lesion before coming to this centre. We have carried out re-excision of the scar tissue and tumour bed in these patients. In 20 out of these 24 (83.3\%) patients, residual tumour was identified in the re-

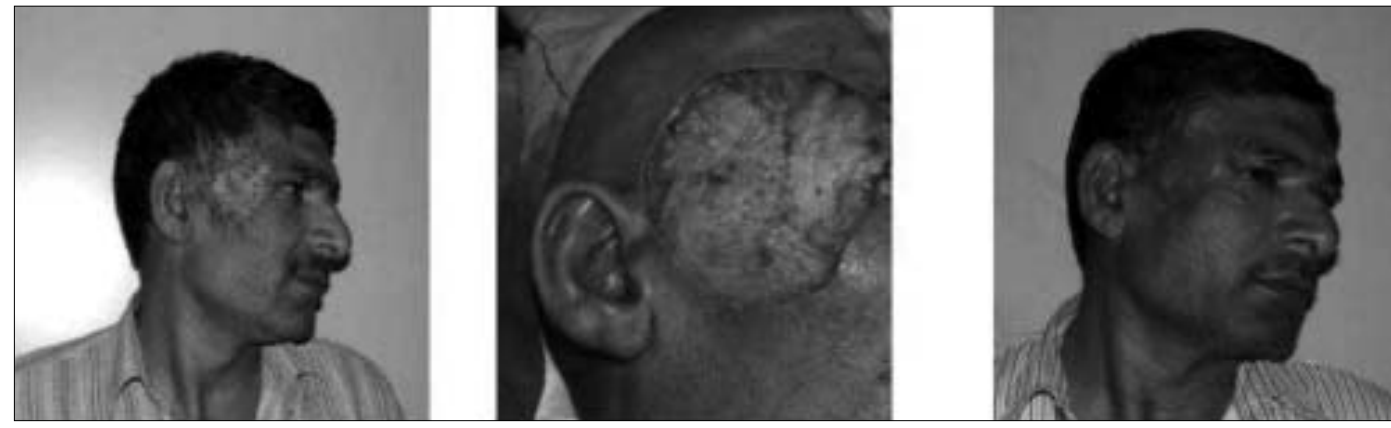

Figure 2: (A) Large superficial BCC involving facial skin. (B) Postresection defect. (C) Late postoperative picture following resection and skin graft

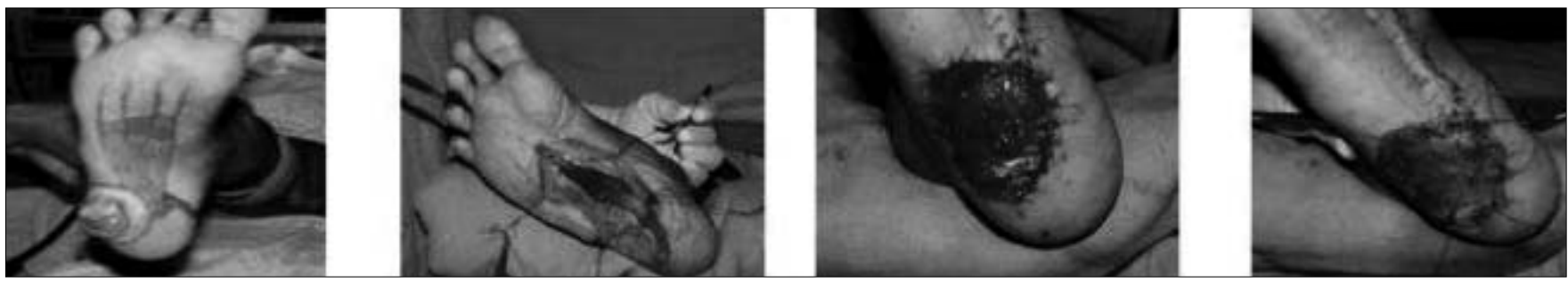

Figure 3: (A) Melanoma of sole. (B) Postresection defect with Flexor digitorum brevis muscle flap. (C) Resection defect covered with muscle flap. (D) Split skin graft over the muscle flap 
excision specimen. This suggests that optimal surgical margin was compromised during the initial surgery at primary care canters. The possible factors for inadequate resection could be - lack of awareness and temptation to achieve a primary closure of defect owing to the lack of reconstruction expertise.

Treatment strategies differ for these three groups. Although, surgery is the mainstay of treatment for all the three common skin cancers, the extent of surgery, both local and regional varies. Adequate surgery is most important to prevent recurrence and long-term cure rates for patients with recurrent lesions are significantly lower than those for patients with primary lesions. Also, recurrent skin cancer is more likely to metastasize. ${ }^{[18]}$ Adequate surgical margin is very important, particularly for melanoma, where margin depends upon the thickness (depth of infiltration) of cancer. ${ }^{[19]}$ Although, various treatment options have been advocated for NMSC, no randomised, prospective study has compared treatment methods. ${ }^{[20]}$

Simple surgical excision is effective for all types of BCCs. The cure rate approaches $99 \%$ when the histological margins are clear. Recommended margin is $5 \mathrm{~mm}$; recurrence is more when the margin of resection is less than $4 \mathrm{~mm} \cdot{ }^{[15,21]}$ Moh's micrographic surgery has been implied for recurrent lesions or those located in vital areas such as eyelid, digits, penis, nose, etc., but it requires a dedicated surgeon pathologist and onsite facility for pathology examination which is not present is most of the centres. For SCC, including locally advanced and recurrent tumours surgical excision is the mainstay of treatment. ${ }^{[15]}$ At least a $1 \mathrm{~cm}$ margin beyond the apparent limit of the tumour is recommended. With the approach of taking proper recommended margins, we could achieve good local control for both BCC and SCC. In the current study, none of the patients with BCC developed local recurrence, whereas only one patient with SCC had local relapse. Regional lymph node dissection has no role in BCC, but in the presence of positive regional lymph nodes, therapeutic lymph node dissection is indicated for SCC. ${ }^{[7]}$ We have carried out regional lymph node dissection in 14 patients with SCC.

The mainstay of treatment for melanoma is surgical excision, with margins being determined by the depth of the tumour. Excision should include skin, subcutaneous tissue up to the muscle fascia. ${ }^{[22]}$ Various trials have addressed the issue of margin. ${ }^{[1]}$ The results of these studies suggest that a $1-\mathrm{cm}$ radial margin is adequate for primaries with thickness up to $1 \mathrm{~mm}$ and $2 \mathrm{~cm}$ margin is adequate for primaries up to $4 \mathrm{~mm}$ thickness. None of these studies have shown that wider margins are necessary or related to improvement in survival. Although patients with primaries more than $4 \mathrm{~mm}$ thickness have a relatively high (approximately13\%) risk of local recurrence, there are few data to support the use of margins wider than $2 \mathrm{~cm} .{ }^{[22]}$ In this study, margin of excision taken for melanoma was $2 \mathrm{~cm}$ because in most of these patients either the thickness of tumour was unknown (if excision biopsy was done elsewhere) or thickness was more than $1 \mathrm{~mm}$. We could achieve good local control, with this strategy and only one out of 20 patients with melanoma developed local recurrence. In addition, control of regional spread is an important part of surgery for melanoma because it has a high propensity for early regional nodal spread. ${ }^{[19]}$ In the current study, 11 out of 20 patients $(55 \%)$ with melanoma underwent regional lymph nodal dissection and all had tumour detected in the nodal specimens. We could not perform sentinel node study routinely because most cases were either recurrent, previously inadequately treated or clinically node positive.

Surgical margin and possibility of requirement of reconstruction are directly related to each other and there is always a critical trade off between them. Any compromise of the adequacy of surgical margin increases the chances of recurrence. A reconstructive procedure is always preferred to a potentially suboptimal surgical excision. ${ }^{[23]}$ We had a liberal approach towards reconstruction of the surgical defects. We have carried out some form of reconstruction in $55.8 \%$ of the patients. The primary defects were closed using skin grafts in $34.88 \%(15 / 43)$ patients and loco regional flaps were used in $65.12 \%(28 / 43)$ patients. Only three patients out of 77 had positive tumour margins. Hence, we feel that reconstruction expertise is necessary for proper management of skin cancers, particularly for locally advanced growths.

The role of adjuvant therapy is limited in skin cancers. Although radiotherapy can be used as primary mode of treatment for BCC and SCC located at certain sites such as the nose, lip, eyelid and canthus, where surgery is either technically difficult or likely to yield poor cosmesis. Radiotherapy has a very limited role in the management of melanoma. ${ }^{[7],[24]}$ Postoperative radiotherapy is indicated in patients with advanced lesions, positive margins, lymph node metastasis, intransit metastases in melanoma and for palliation. ${ }^{[25]}$ In this study, adjuvant radiotherapy to local and/or regional sites was given in $27.2 \%$ patients for the indications mentioned earlier. Adjuvant chemotherapy and immunotherapy is generally used in advanced stage melanoma but results show only limited success..$^{[9,26]}$ 
None of the patients in this study received chemotherapy or immunotherapy.

In the current study, the rate of loco-regional disease relapse was $12.9 \%$ and 5 -year median disease-free survival in the study population was $75 \%$. These results suggest that it is possible to achieve reasonably good loco-regional control of disease with optimal surgery taking adequate margins and a reconstructive surgical procedure when needed.

\section{Conclusions}

Skin cancers constitute a small but significant proportion of patients with cancer. Unlike in the Western countries, SCC is the commonest histologic variety. Primary level inadequate intervention is very common. Good results can be obtained with radical surgery and optimal surgical margins along with a reconstructive procedure when needed.

\section{References}

1. WHO World health statistics. GLOBOCAN 2000: Cancer Incidence, Mortality and Prevalence Worldwide. Version 1.0. IARC CancerBase No. 5. Lyon, IARC Press; 2001.

2. Howe HL, Wingo PA, Thun MJ, Ries LAG, Rosenberg HM, Feigal $E G$, et al. Annual report to the nation on the status of cancer (1973 through 1998), featuring cancers with recent increasing trends. J Natl Cancer Inst 2001;93:824-42.

3. Godbole VK, Toprani HT, Shah HH. Skin cancer in Saurashtra. Ind J Pathol Bacteriol 1968;11:183-9.

4. National Cancer Registry Programme, Indian Council of Medical Research. Consolidated report of the population based cancer registries 1990-96.

5. Khanolkar VR, Suryabhai B. Cancer in relation to usages. Arch Path 1945;40:351-61.

6. Nair MK, Varghese C, Mahadevan S, Cherian T, Joseph F. Cutaneous malignant melanoma- clinical epidemiology and survival. J Indian Med Assoc 1998;96:19-20.

7. Mahajan MK, Lal P, Biswal BM, Mohanti BK. Cancer of the skin. In: Rath GK, Mohanti BK, editors. Textbook of Radiation Oncology: Principles and Practice. New York: B. I. Churchill Livingstone; 2000. p. 223-37.

8. Krishnamurthy S, Yeole B, Joshi S, Gujarathi M, Jussawalla DJ. The descriptive epidemiology and trends in incidence of nonocular malignant melanoma in Bombay and India. Indian J Cancer 1994;31:64-71.

9. Doll R. Are we winning the fight against cancer? An epidemiologic assessment. EACR- Muhlbock memorial lecture. Eur J Can 1990;26:500-8.

10. Shapiro RL. Surgical approaches to malignant melanoma, practical guidelines. Dermatol Clin 2002;20:681-99.

11. Essner R. Surgical treatment of malignant melanoma. Surg Clin N Am 2003;83:109-56.

12. Budhraja SN, Pillai VC, Periyanayagam WJ, Kaushik SP, Bedi BM. Malignant neoplasms of the skin in Pondicherry. Indian J Cancer 1972;9:284-95.

13. Chuang TY, Popescu NA, Su WP, Chute CG. Squamous cell carcinoma: a population-based incidence study in Rochester, Minn. Arch Dermatol 1990;126:185-8.

14. Reintgen DS, McCarty KS, Woodard B, Cox E, Seigler HF. Metastatic malignant melanoma from an unknown primary. Ann Surg 1983; 156:335-50.

15. Gendleman MD, Victor TA, Tsitsis T. Nonmelanoma skin cancer. In: Winchester DP, Jones RS, Murphy GP, editors. Cancer Surgery for the general Surgeon. New York: Lippincott Williams \& Wilkins; 1999. p. 111-35.

16. Vijaykumar DK, Kanan RR, Chaturvedi HK. Plantar acral melanoma- an experience from a regional cancer center, India. Indian J Cancer 1996;33:122-9.

17. White WL, Loggie BW. Sentinel lymphadenectomy in the management of primary cutaneous malignant melanoma: an update. Dermatol Clin 1999;17:645-55.

18. Rowe DE, Caroll RJ, Day CL Jr. Prognostic factors for local recurrence, metastasis, and survival rates in Squamous cell carcinoma of the skin, ear, and lip: implications for treatment modality selection. J Am Acad dermatol 1992;26:976-90.

19. Morton DL, Wanek L, Nizze JA, Elashoff RM, Wong JH. Improved long-term survival after lymphadenectomy of malignant melanoma metastatic to the regional lymph nodes. Ann Surg. 1991;214:491.

20. Preston DS, stern RS. Nonmelanoma cancers of the skin. N Engl J Med 1992;327:1649-62.

21. Goldberg LH. Basal cell carcinoma [Review]. Lancet 1996; 347:663.

22. Kenady DE, Brown BW, Mc Bride CM. Excision of underlying fascia with a primary malignant melanoma: effect on recurrence and survival rates. Surgery 1982;92:615-8.

23. Aalst JAV, McCurry T, Wagner J. Reconstructive considerations in the surgical management of melanoma. Surg Clin N Am 2003;83: 187-230.

24. Goldschimidt H, Breneman JC, Breneman DL. Ionizing radiation therapy in dermatology [Review]. J Am Acad dermatol 994;30: 157.

25. Vora SA, Garner SL. Role of radiation therapy for facial skin cancers. Clin Plast Surg 2004;31:33-8.

26. Yeung RS. Management of recurrent cutaneous melanoma. Curr Probl Cancer 1994; 14:143. 